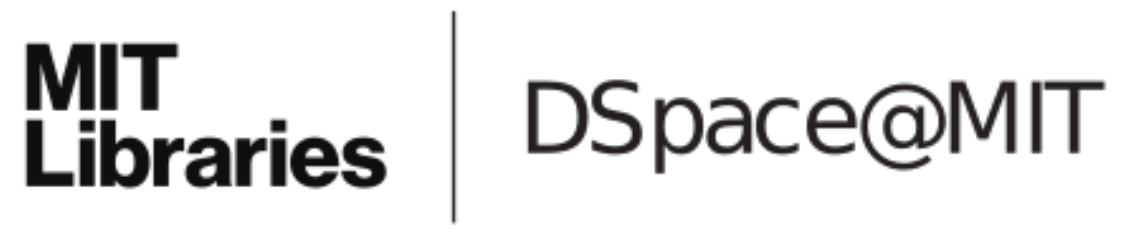

\author{
MIT Open Access Articles
}

\section{Resonant cavity enhancement of polycrystalline PbTe films for IR detectors on Si-ROICs}

The MIT Faculty has made this article openly available. Please share how this access benefits you. Your story matters.

Citation: Wang, Jianfei, Timothy Zens, Juejun Hu, Piotr Becla, Anuradha M. Agarwal, and Lionel C. Kimerling. "Resonant cavity enhancement of polycrystalline PbTe films for IR detectors on SiROICs." Proc. SPIE 8034, Photonic Microdevices/Microstructures for Sensing III, 80340K (May 16, 2011). (C) (2011) COPYRIGHT Society of Photo-Optical Instrumentation Engineers (SPIE)

As Published: http://dx.doi.org/10.1117/12.884081

Publisher: SPIE

Persistent URL: http://hdl.handle.net/1721.1/79717

Version: Final published version: final published article, as it appeared in a journal, conference proceedings, or other formally published context

Terms of Use: Article is made available in accordance with the publisher's policy and may be subject to US copyright law. Please refer to the publisher's site for terms of use. 


\title{
Resonant cavity enhancement of polycrystalline PbTe films for IR detectors on Si-ROICs
}

\author{
Jianfei Wang, Timothy Zens, Juejun Hu, Piotr Becla, Anuradha M. Agarwal, and Lionel C. \\ Kimerling \\ Microphotonics Center, Massachusetts Institute of Technology, Cambridge, MA 02139, USA
}

\begin{abstract}
In this paper, we demonstrate high optical quantum efficiency (90\%) resonant-cavity-enhanced mid-infrared photodetectors fabricated monolithically on a silicon platform. High quality photoconductive polycrystalline $\mathrm{PbTe}$ film is thermally evaporated, oxygen-sensitized at room temperature and acts as the infrared absorber. The cavity-enhanced detector operates in the critical coupling regime and shows a peak responsivity of $100 \mathrm{~V} / \mathrm{W}$ at the resonant wavelength of $3.5 \mu \mathrm{m}, 13.4$ times higher compared to blanket PbTe film of the same thickness. Detectivity as high as $0.72 \times 10^{9}$ $\mathrm{cmHz}^{1 / 2} \mathrm{~W}^{1}$ has been measured, comparable with commercial polycrystalline mid-infrared photodetectors. As low temperature processing $\left(<160^{\circ} \mathrm{C}\right)$ is implemented in the entire fabrication process, our detector is promising for monolithic integration with $\mathrm{Si}$ readout integrated circuits.
\end{abstract}

Keywords: PbTe, nanocrystalline, Infrared, MWIR, microcavity

\section{Introduction}

High-performance photodetectors and imagers operating in the mid-infrared (mid-IR) wavelength range (3-10 $\mu \mathrm{m})$ is attracting increasing interest recently due to their wide applications in security surveillance, chemical sensing, and industrial process monitoring [1]. Currently, most mid-IR detectors rely on single-crystalline compound semiconductors such as HgCdTe and III-V ternary alloys for infrared absorption. While detectors and focal plane arrays (FPAs) based on these exotic alloys feature high quantum efficiency and responsivity, the FPAs have to be flip-chip bonded onto silicon readout integrated circuits (Si ROIC) for signal readout and processing. Thermal expansion coefficient mismatch and poor mechanical stability often lead to low yield of such hybrid devices. The high-cost single crystal compound semiconductor deposition and processing involved in the fabrication also make mid-IR FPAs prohibitively expensive. Further, typical mid-IR detectors require a large absorber layer thickness in the order of $10 \mu \mathrm{m}$ for high quantum efficiency, dictated by the relatively low optical absorption coefficient of these semiconductors at mid-IR. On the other hand, polycrystalline lead salts show promise in greatly reducing the cost for mid-IR detection. Uncooled FPA of polycrystalline $\mathrm{PbSe}$ has been developed recently and detectivity up to $3 \times 10^{9} \mathrm{cmHz}^{1 / 2} \mathrm{~W}^{1}$ has been achieved [2]. Here we present a demonstration of mid-IR detectors monolithically integrated on silicon using nanocrystalline PbTe as the IR active material. The entire detector structure can be directly deposited onto a silicon substrate free of lattice match constraints. While it was previously believed that high-temperature sensitization is required for polycrystalline lead salt films to become IR sensitive [3,4], our nanocrystalline PbTe films show excellent IR response through a roomtemperature oxygen sensitization process. The electrical transport properties and photoconductivity in visible spectral range have been extensively studied by Dobrovolsky and Dashevsky [5,6]. Details of the PbTe material development and characterizations are outside the scope of this paper and will be published elsewhere [7]. To resolve the fabrication challenge associated with thick IR absorber deposition, we employ a resonant-cavity-enhanced (RCE) design to maintain nearunity quantum efficiency at a much decreased absorber thickness. Since most types of noise including generationrecombination noise, shot noise and Johnson noise scale with the absorber volume, the small absorber thickness in an RCE design also leads to reduced detector noise [8]. RCE photodetectors for mid-IR have been demonstrated in singlecrystalline $\mathrm{PbTe}$ [9], $\mathrm{Pb}_{1-\mathrm{x}} \mathrm{Eu}_{\mathrm{x}} \mathrm{Se}$ [10], and $\mathrm{HgCdTe}$ [11]. Our approach, which uses polycrystalline and amorphous materials for cavity fabrication, not only reduces the cost and allows monolithic integration with Si ROICs, but also enables simultaneous multispectral detection within a single RCE detector pixel for chemical-bio sensing and hyperspectral imaging applications $[12,13]$.

\section{Photodetector design}

Quantum efficiency in this work is defined as the ratio of the number of photon generated excess electron-hole pairs over the number of incident photons, and will be denoted as $\mathrm{QE}$. Due to the fact that PbTe is direct band gap semiconductor,

Photonic Microdevices/Microstructures for Sensing III,

edited by Hai Xiao, Xudong Fan, Anbo Wang, Proc. of SPIE Vol. 8034 ,

80340K - (c) 2011 SPIE · CCC code: 0277-786X/11/\$18 - doi: 10.1117/12.884081

Proc. of SPIE Vol. $803480340 K-1$ 
we have made the assumption that all absorbed photons contribute to generating electron-hole pairs. The key design challenge for RCE detectors is how to attain critical coupling, the prerequisite of achieving near unity quantum efficiency. In a planar cavity sandwiched between two mirrors, the critical coupling condition can be generically written as:

$$
\left.Q_{t m}=Q_{a b s} \text { and } Q_{b m} \rightarrow \infty \text { (i.e. } R_{b m} \rightarrow 1\right)
$$

where $\mathrm{Q}_{\mathrm{tm}}, \mathrm{Q}_{\mathrm{bm}}$, and $\mathrm{Q}_{\mathrm{abs}}$ represent the cavity quality factors $(\mathrm{Q})$ due to top mirror loss, bottom mirror loss, and absorption of the IR active layer, and $\mathrm{R}_{\mathrm{bm}}$ is the bottom mirror reflectance. Notably, when the IR active material completely fills the cavity, the formula can be reduced to Unlu's formulation [8]. However, as using a thin active layer contributes to reduced noise, we resort to a general formulation applicable to a "sandwiched" cavity configuration (Fig. 1). Perturbation theory gives $Q_{a b s}$ as:

$$
Q_{a b s}=\frac{4 \pi}{\lambda n \cdot \alpha} \frac{\int_{\text {stack }} d r \cdot \varepsilon_{c}\left|E_{0}\right|^{2}}{\int_{\text {active }} d r \cdot\left|E_{0}\right|^{2}} \approx \frac{4 \pi}{\lambda n \cdot \alpha d} \frac{\int_{\text {stack }} d r \cdot \varepsilon_{c}\left|E_{0}\right|^{2}}{\left|E_{0, \text { active }}\right|^{2}}
$$

where $\mathrm{n}, \alpha$ and $\mathrm{d}$ are the index of refraction, absorption coefficient and thickness of the IR active material, $\lambda$ is the resonant wavelength, E0 denotes the electric field distribution of the resonant mode, and $\varepsilon \mathrm{c}$ gives the relative dielectric constant profile of the stack. The second equality in Eq. (1) holds when $\lambda>>d$, in which case the electric field can be treated as a constant in the IR active layer ( $\mathrm{E}_{0}$, active). Since $\mathrm{Q}_{\mathrm{tm}}$ is determined by the top mirror reflectance Rtm, Eq. (1) states that given a bottom mirror with near unity reflectance $\left(\mathrm{R}_{\mathrm{bm}} \rightarrow 1\right)$, maximum $\mathrm{QE}$ at resonant wavelength may be obtained by appropriately choosing a top mirror reflectance to satisfy Eq. (1). To illustrate the principle of critical coupling, Fig. 2 plots quantum efficiency in an RCE detector as a function of $\mathrm{Q}_{t m}$ given $\mathrm{R}_{\mathrm{bm}} \rightarrow 1$. The $\mathrm{Q}_{\mathrm{abs}}$ value of the designed RCE detector structure is derived to be 85 using Eq. (2) and fixed as a constant in the calculation. The calculation employs a modified two-port coupling matrix approach [14]. Clearly, unity $\mathrm{QE}$ is attained only when $\mathrm{Q}_{\mathrm{tm}}=$ $\mathrm{Q}_{\mathrm{abs}}$, in agreement with the condition specified by Eq. (1).

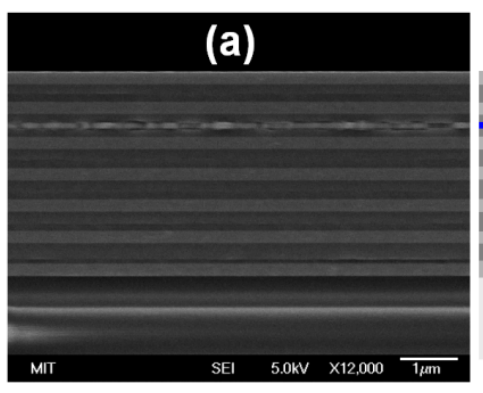

(b)

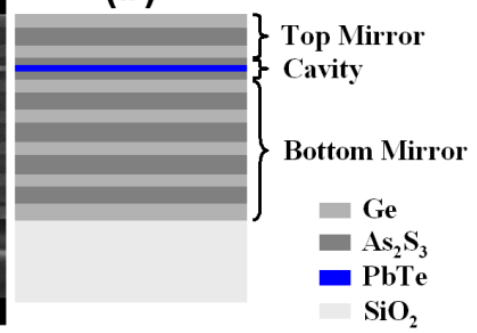

Fig. 1. (a) SEM cross-sectional image of the resonant cavity structure deposited onto a $\mathrm{SiO}_{2} / \mathrm{Si}$

substrate. (b) Schematic picture of the designed structure. All fifteen layers are clearly seen in (a), demonstrating good film thickness control and uniformity. Sharp interfaces between different layers indicate negligible material inter-diffusion occurs since processing temperatures are low $\left(<160^{\circ} \mathrm{C}\right)$. 


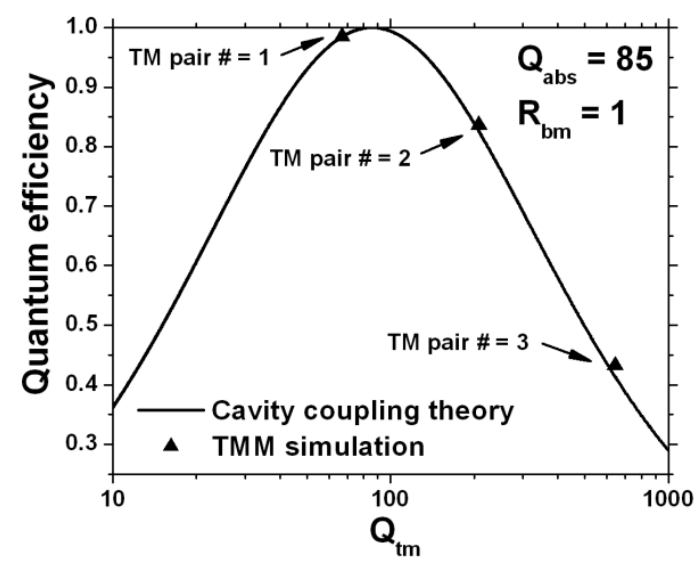

Fig. 2. QE of RCE detector plotted as a function of $\mathrm{Q}_{\mathrm{tm}}$ calculated using a modified coupling matrix method; the triangles correspond to TMM simulated QE values of designs with different top mirror (TM) Bragg pair numbers. Near unity QE is attained under critical coupling condition, i.e. $\mathrm{Q}_{\mathrm{tm}}=\mathrm{Q}_{\mathrm{abs}}$.

To experimentally demonstrate near unity $\mathrm{QE}$, we tune the top mirror reflectance and $\mathrm{Q}_{\mathrm{tm}}$ by using a top mirror consisting of an appropriate number of Bragg pairs to satisfy Eq. (1). $\mathrm{As}_{2} \mathrm{~S}_{3}$ and Ge are used as the Bragg mirror materials given their IR transparency. Their wavelength-dependent refractive indices are calculated from experimentally measured transmittance spectra using the Swanepoel approach [15]. Refractive indices of $\mathrm{As}_{2} \mathrm{~S}_{3}$ and Ge used to calculate the Bragg pairs are 2.363 and 4.105 respectively around $3.5 \mu \mathrm{m}$ wavelength. The refractive indices of PbTe IR absorber are measured using IR ellipsometry [16]. Using the experimentally measured indices as input and a $100 \mathrm{~nm}$ thick PbTe layer as the IR active material, QE of RCE detector designs with different top mirror $\mathrm{As}_{2} \mathrm{~S}_{3}$-Ge Bragg pair numbers may be simulated using the transfer matrix method (TMM). The TMM simulated QE values

are represented in Fig. 2 as the triangles; the excellent agreement between the modified coupling matrix theory and TMM confirms that the coupling matrix method conventionally employed for micro-ring resonator simulations may also be applied for quantitative prediction of QE in RCE detectors.

\section{Experiment}

All films are deposited in the same chamber. 2" diameter poly-Ge of $99.999 \%$ purity is used as sputtering target, $\mathrm{As}_{2} \mathrm{~S}_{3}$ and $\mathrm{PbTe}$ bulk of $99.999 \%$ purity are used as the source material for thermal evaporation. Both sputtering and thermal evaporation are carried out at a base pressure of $5 \times 10^{7}$ Torr. Optically polished $\mathrm{CaF}_{2}$ discs and oxide coated $\mathrm{Si}$ wafers are used as starting substrates. The substrates are held on a rotating substrate holder, at room temperature, throughout the depositions. Film deposition rate is monitored in real-time through a quartz crystal sensor and is maintained at $1.3 \AA / \mathrm{s}$ for $\mathrm{Ge}$, and $\sim 10 \AA / \mathrm{s}$ for $\mathrm{As}_{2} \mathrm{~S}_{3}$ and $\mathrm{PbTe}$. Photodetectors are fabricated by a three-step lift-off process. The alignment is done using a Karl Suss Model MJB-3 Mask Aligner with a $350 \mathrm{~W} \mathrm{Hg}$ bulb. Negative photoresist is exposed to $320 \mathrm{~nm}$ UV light and developed to make openings for film deposition, which is followed by lift-off process in an ultrasonic bath [17]. Three lift-off steps are used to pattern the bottom mirror and $\mathrm{PbTe}$, top mirror, and $\mathrm{Sn}$ contacts separately. To measure photoconductivity of the fabricated detectors, light from a $100 \mathrm{~W}$ quartz tungsten halogen (QTH) lamp is modulated at $98.0 \mathrm{~Hz}$ and monochromatized by a Newport Oriel MS257 monochromator. Long-pass optical filters are used to block higher order diffracted light. A pyroelectric detector with known photo-responsivity is used as a reference detector to obtain the power density of light incident onto the samples. A thermoelectric cooler (TEC) is used to cool samples down to $56^{\circ} \mathrm{C}$ and a Keithley 6220 current source is used to provide bias current.

\section{Results}

Figure 1(a) shows the cross-sectional SEM image of the resonant cavity structure deposited onto a $\mathrm{SiO}_{2} / \mathrm{Si}$ substrate, and for comparison, Fig. 1(b) shows a schematic picture of the designed RCE detector structure. All fifteen layers are clearly identified in the SEM image, illustrating good film thickness control and uniformity. Sharp interfaces between different layers indicate negligible material inter-diffusion occurs since processing temperatures are low $\left(<160^{\circ} \mathrm{C}\right)$. The cavity uses $100 \mathrm{~nm}$ thick PbTe as the absorber. In comparison, a conventional freespace PbTe photodetector operating at 3.5 
$\mu \mathrm{m}$ wavelength requires an absorber layer as thick as $10 \mu \mathrm{m}$. A consequence of the thin PbTe detector layer $(100 \mathrm{~nm})$ is that the generationrecombination and Johnson noise are reduced by one order of magnitude, since both types of noises scale with the square root of the active material volume. A comparison (Fig. 3) of the reflectance spectra of the cavity obtained experimentally by FTIR and theoretically by TMM simulation shows excellent agreement. The spectra feature a photonic band gap (PBG) from $2.5 \mu \mathrm{m}$ to $4.5 \mu \mathrm{m}$ and a resonant cavity mode at $3.5 \mu \mathrm{m}$, as designed. Figure 3 shows only first order cavity mode since here we use a half wavelength cavity and thus it does not support higher order cavity modes. At the resonant wavelength of $3.5 \mu \mathrm{m}$, directly measured reflectance and transmittance values are $6.6 \%$ and $3.5 \%$ respectively, indicating a quantum efficiency of $90 \%$. Full width at half maximum (FWHM) is $92.53 \mathrm{~nm}$, corresponding to a quality factor of 38 , in good agreement with the simulated value of $43\left(=0.5 \mathrm{Q}_{\text {abs }}\right)$.

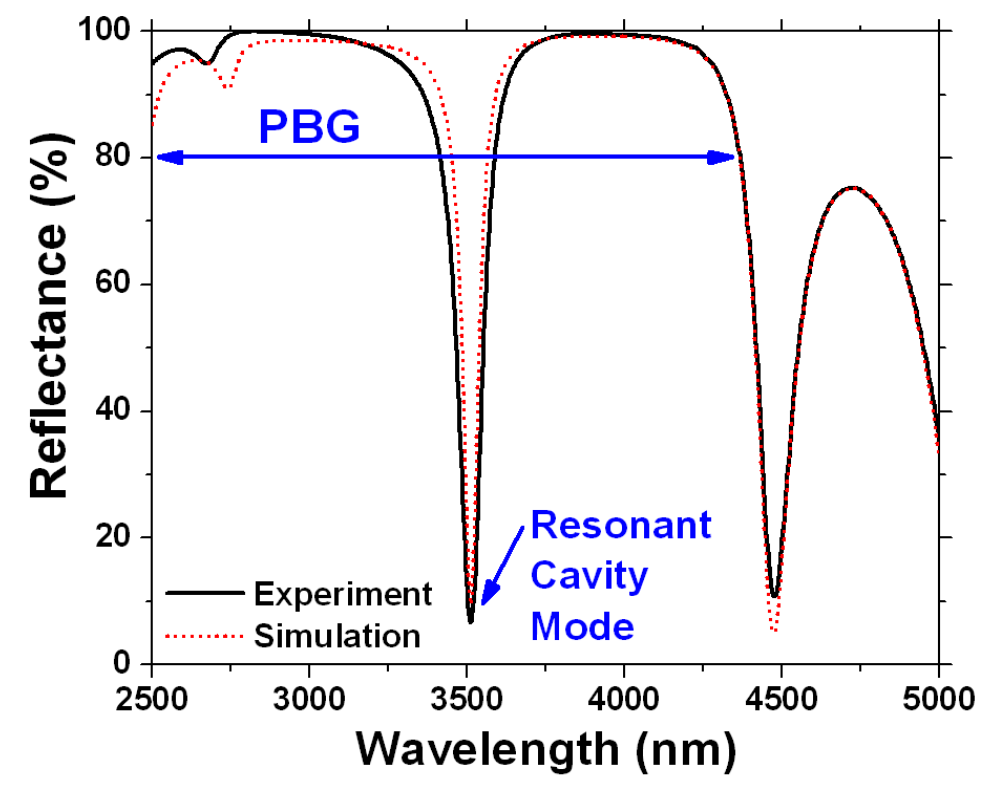

Fig. 3. Reflectance spectra of the cavity structure obtained by FTIR measurement (solid line) and TMM simulation (dotted line), showing excellent agreement. The spectra feature a PBG from $2.5 \mu \mathrm{m}$ to $4.5 \mu \mathrm{m}$ and a resonant cavity mode at $3.5 \mu \mathrm{m}$ as designed.

Room temperature Hall measurement shows p-type conduction in the PbTe absorbing layer with a Hall mobility of 99 $\mathrm{cm}^{2} \mathrm{~V}^{1} \mathrm{~s}^{1}$ and a carrier concentration of $4.1 \times 10^{16} \mathrm{~cm}^{-3}$. This is consistent with our PbTe plain film results [7] and indicates no film quality degradation has occurred during the photodetector fabrication. Figure 4 demonstrates the operation of the polycrystalline PbTe based RCE photodetector. In the photoconductivity measurement samples are biased under fixed current, and the photovoltage caused by the IR light illumination is recorded. Photon incident flux and spectral shape are obtained from the pyroelectric reference detector with known area and responsivity. The inserted picture on the right shows the electric circuit used. Peak responsivity of $100 \mathrm{~V} / \mathrm{W}$ is measured at the resonant wavelength of $3.45 \mu \mathrm{m}$. The small discrepancy $(<2 \%)$ of the resonant wavelength location between Fig. 3 and Fig. 4 is due to nonnormal incidence of light in both experiments. The wavelength resolution in the photoconductivity measurement is $\sim 45$ $\mathrm{nm}$ in the wavelength range of interest. To quantify the resonant cavity enhancement effect, $\mathrm{PbTe}$ plain film photodetector of the same thickness and dimensions has been measured under the same conditions as shown in Fig. 4. $100 \mathrm{~nm}$ thick PbTe plain film shows much lower photoresponse $(<10 \mathrm{~V} / \mathrm{W})$ in mid-IR range due to very weak light absorption, while $\mathrm{PbTe}$ in the cavity shows 13.4 times higher responsivity at the designed resonant wavelength. 


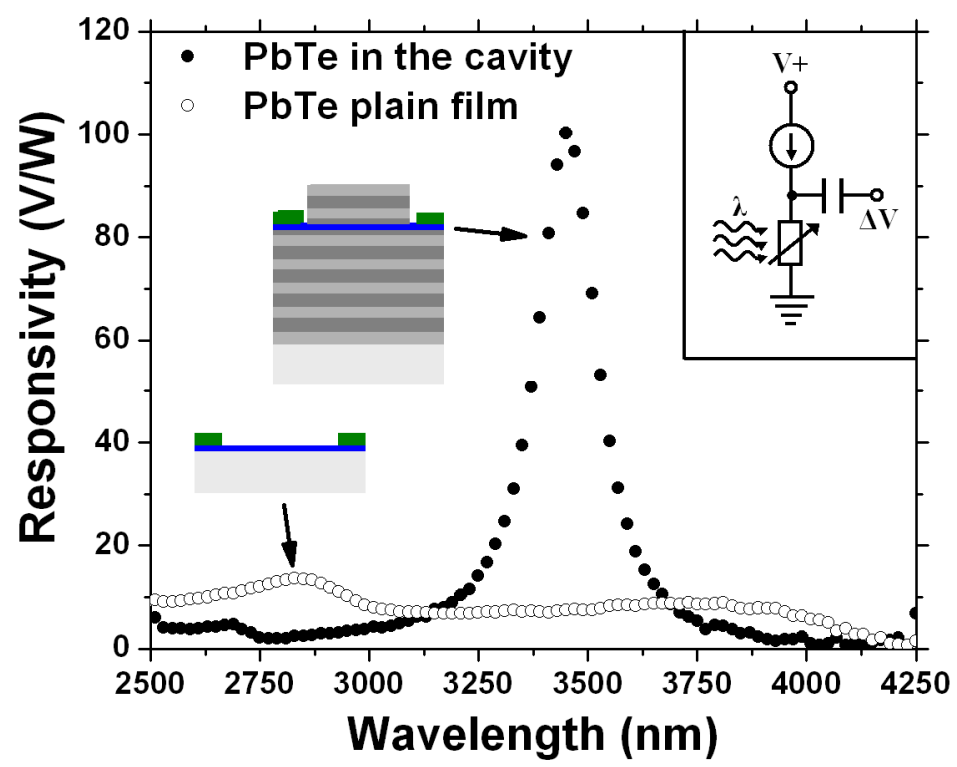

Fig. 4. Responsivity spectra of the $100 \mathrm{~nm}$ PbTe thin film photodetector within the cavity (filled circles) and without cavity (plain film only, open circles) with $0.1 \mathrm{~mA}$ bias current. The two inserted pictures on the left show the tested device structures schematically after metal deposition and patterning (green colored regions). The inserted picture on the right shows the electric circuit employed in the photoconductivity measurement. Plain film shows much lower photoresponse $(<10 \mathrm{~V} / \mathrm{W})$ in mid-IR range due to very weak light absorption, while $\mathrm{PbTe}$ in the cavity shows 13.4 times higher responsivity at the designed resonant wavelength.

Analysis of noise mechanisms including background, generation-recombination, and Johnson noises in the photoconductor identifies Johnson noise is the dominant noise. Johnson noise current can be measured by:

$I_{J}=\sqrt{\frac{4 k T \Delta f}{R}}$

where $\mathrm{k}$ is Boltzmann constant, $\mathrm{T}$ is absolute temperature, $\Delta \mathrm{f}$ is frequency bandwidth, and $\mathrm{R}$ is photodetector's resistance value in dark space. Thus Johnson-noise-limited detectivity $\left(\mathrm{D}^{*}\right)$ can be measured by:

$D^{*}=\frac{(\text { Responsivity } / R) \sqrt{A \Delta f}}{I_{J}}$

from Responsivity and Johnson noise current measured as described before, and photodetector's area (A). On the other hand, expression of Responsivity can be derived from the carrier number modulation model:

Responsivity $=\frac{I(1+b) Q E \tau \lambda}{h c e w^{2} d^{2} p^{2} \mu_{p}}$

where I is bias current, $\mathrm{b}$ is the mobility ratio $\left(\mu_{\mathrm{e}} / \mu_{\mathrm{p}}\right), \mathrm{QE}$ is the quantum efficiency, $\tau$ is carrier lifetime, $\lambda$ is wavelength, $\mathrm{w}$ is width of the photodetector, $\mathrm{d}$ is thickness of the photoactive layer, $\mathrm{p}$ is holes' concentration, $\mu_{\mathrm{p}}$ is holes' mobility, and h, c, and e are Plank constant, speed of light in vacuum, and elementary charge respectively. Substitute Eqs. (3) and (5) into Eq. (4), one obtains:

$D^{*}=\frac{I(1+b) Q E \tau \lambda}{h c w \sqrt{4 k T e d^{3} p^{3} \mu_{p}}}$

Notably, even though detectivity is typically independent of detector size when the device operates in the photovoltaic mode, Eq. (6) suggests Johnson-noise-limited detectivity in a photoconductive detector is inversely proportional to the 
linear dimension of the photodetector. Thus higher detectivity values can be expected for photodetectors with smaller size. To demonstrate this mechanism, RCE photodetectors of different sizes $(100 \sim 1200 \mu \mathrm{m})$ are tested under the same bias current of $50 \mu \mathrm{A}$. Their peak detectivity values measured according to Eq. (4) are shown as solid squares in Fig. 5. With the known dependence of detectivity on $w$ as described in Eq. (6), the measured data is fitted according to Eq. (6). Excellent agreement between theoretical fit based on Eq. (6) and experimental data can be seen in Fig. 5. Detectivity value up to $0.72 \times 109 \mathrm{cmHz}^{1 / 2} \mathrm{~W}^{1}$ is obtained in a $100 \mu \mathrm{m}$ wide RCE phototector while photoactive layer's thickness is only $100 \mathrm{~nm}$, comparable with commercial polycrystalline mid-IR photodetectors fabricated via chemical bath deposition. Further reduction of photodetector size will lead to higher detectivity value $\left(\sim 10^{10} \mathrm{cmHz}^{1 / 2} \mathrm{~W}^{1}\right)$ as is predicted by Eq. (6).

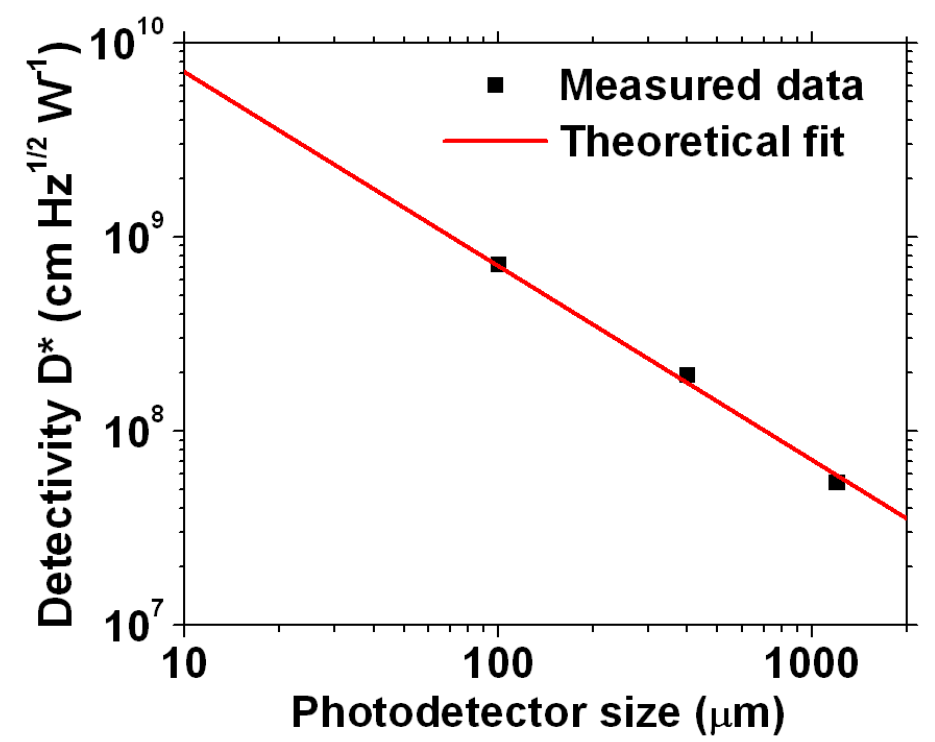

Fig. 5. Detectivity D* as a function of photodetector size with square shape. Theoretically fitted curve is based on Eq. (3) showing the inversely proportional relationship between $\mathrm{D}^{*}$ and photodetector size. Solid squares correspond to experimentally measured data.

Figure 6(b) shows the temperature dependence of SIDAS photodetector resistivity (size $400 \mu \mathrm{m} \times 400 \mu \mathrm{m}$ ), which shows a thermally activated process with an activation energy of $0.155 \mathrm{eV}$.
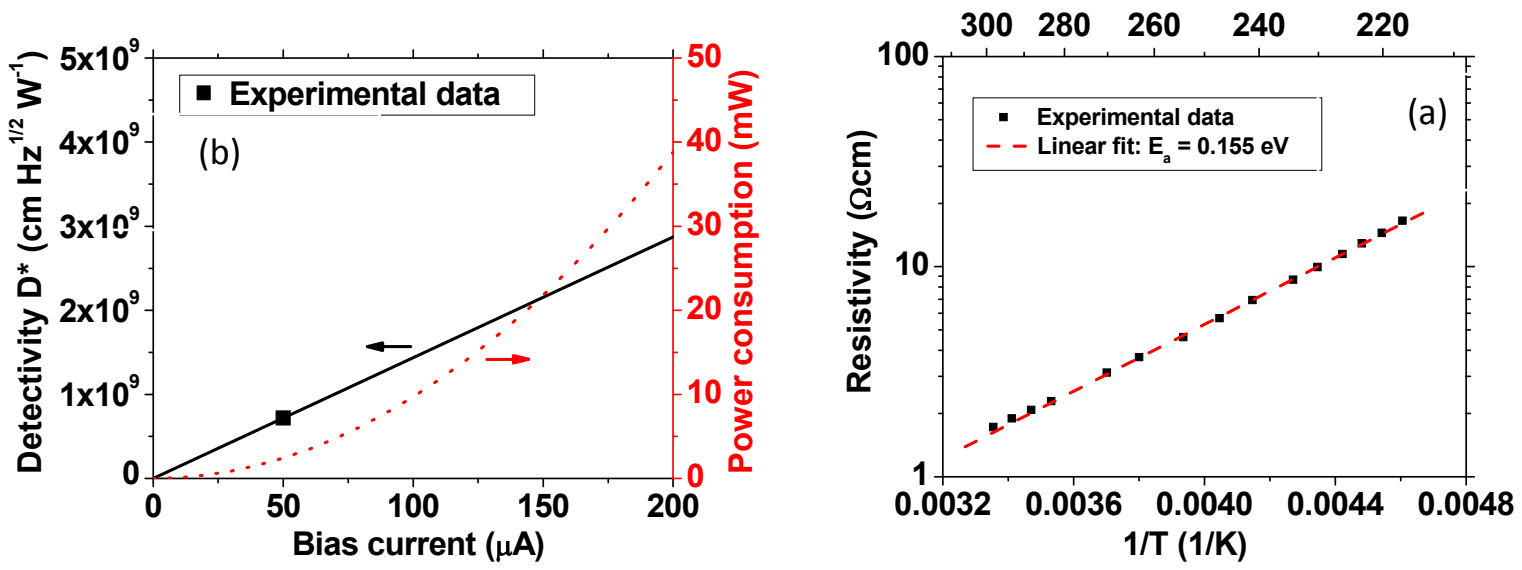

Fig. 6. (a) Photodetector detectivity and power consumption as a function of bias current in a $100 \mu \mathrm{m}$ x $100 \mu \mathrm{m}$ pixel measured at 98 $\mathrm{Hz}$ at a temperature of $-53^{\circ} \mathrm{C}$ with a resistance of $970 \mathrm{k} \Omega$. (b) Photodetector resistivity as a function of temperature in a $400 \mu \mathrm{m} \times 400$ 
$\mu \mathrm{m}$ pixel. The result is consistent with previous work and it shows a thermally activated process with an activation energy of 0.155 $\mathrm{eV}$.

Based on the experimental data in Figure 5 and Eq. 6, Johnson-noise-limited detectivity values at different bias current levels can be calculated, as shown in Figure 6(a). Even though Eq. 6 predicts detectivity increases with bias current obeying a linear relationship without limitation, in reality this trend will stop at certain bias current level due to the Joule heating effect and the increase of related current noise. This is determined by the cooling capability of the TEC and the heat sink underneath removing the heat generated during photodetector operation. With known photodetector resistance value $\mathrm{R}$ and bias current $\mathrm{I}$, the power consumption or the heat generated can be calculated by:

$$
P=I^{2} \cdot R
$$

As shown in Figure 6(a), with $100 \mu \mathrm{A}$ bias current, power consumption of one photodetector is $9.7 \mathrm{~mW}$. For a focal plane array (FPA) with $8 \times 8$ photodetectors, the power consumption is less than $1 \mathrm{~W}$, which is in the acceptable range.

However, for FPA with over one million photodetectors, this will result in a power consumption level of $10 \mathrm{~kW}$ which is excessive. So as shown in Figure 6(a), a trade-off has to be made between the required detectivity value and the power consumption budget and heat dissipation strategy. This will be determined by the real application needs, Si ROIC specs, TEC cooling capability, and specially designed heat sink for faster heat dissipation.

\section{Conclusion}

We design and demonstrate mid-IR RCE photodetectors based on polycrystalline PbTe thin films. The resonant cavity mode around $3.5 \mu \mathrm{m}$ is identified by both optical and photoconductive measurements. Compared with $\mathrm{PbTe}$ plain film photodetector, the cavity structure shows 13.4 times higher photoresponse due to the field enhancement effect. The RCE photodetector described here features high quantum efficiency (90\%) and estimated 10- fold reduced noise; further, our approach enables monolithic integration of dense pixel arrays with silicon ROIC, and are thus promising for high performance mid-IR FPA applications.

\section{Acknowledgments}

This work is supported by DSO National Laboratories, Singapore. The authors would like to thank Microsystems Technology Laboratories (MTL) at MIT for fabrication facilities and the Center for Materials Science and Engineering (CMSE) at MIT for characterization facilities. The authors also acknowledge Dr. Xiaochen Sun for providing the TMM simulation codes.

\section{REFERENCES}

1. A. Rogalski, J. Antoszewski, and L. Faraone, “Third-generation infrared photodetector arrays,” J. Appl. Phys. 105(9), 091101 (2009).

2. M. T. Rodrigo, F. J. Sánchez, M. C. Torquemada, V. Villamayor, G. Vergara, M. Verdú, L. J. Gómez, J. Diezhandino, R. Almazán, P. Rodríguez, J. Plaza, I. Catalán, and M. T. Montojo, "Polycrystalline lead selenide x-y addressed uncooled focal plane arrays," Infrared Phys. Technol. 44(4), 281-287 (2003). 3. T. S. Moss, "Lead salt photoconductors," Proc. IRE 43, 1869-1881 (1955).

4. D. E. Bode, T. H. Johnson, and B. N. McLean, "Lead selenide detectors for intermediate temperature operation," Appl. Opt. 4(3), 327-331 (1965).

5. A. A. Dobrovolsky, Z. M. Dashevsky, V. A. Kasiyan, L. I. Ryabova, and D. R. Khokhlov, "Photoconductivity of oxidized nanostructured PbTe(In) films," Semicond. Sci. Technol. 24(7), 075010 (2009).

6. Z. Dashevsky, E. Shufer, V. Kasiyan, E. Flitsiyan, and L. Chernyak, "Influence of oxygen treatment on transport 
properties of PbTe:In polycrystalline films," Physica B 405(10), 2380-2384 (2010).

7. J. Wang, J. Hu, P. Becla, A. Agarwal, and L. C. Kimerling, Microphotonics Center, Massachusetts Institute of Technology, Cambridge, MA 02139, are preparing a manuscript to be called "Highly textured, roomtemperaturesensitized nanocrystalline PbTe film on silicon for infrared detection."

8. M. S. Unlu, and S. Strite, “Resonant cavity enhanced photonic devices,” J. Appl. Phys. 78(2), 607-639 (1995).

9. M. Boberl, T. Fromherz, T. Schwarzl, G. Springholz, and W. Heiss, "IV-VI resonant-cavity enhanced photodetectors for the mid-infrared," Semicond. Sci. Technol. 19(12), L115-L117 (2004).

10. M. Arnold, D. Zimin, and H. Zogg, "Resonant-cavity-enhanced photodetectors for the mid-infrared," Appl. Phys. Lett. 87(14), 141103 (2005).

11. J. G. A. Wehner, C. A. Musca, R. H. Sewell, J. M. Dell, and L. Faraone, "Mercury cadmium telluride resonantcavityenhanced photoconductive infrared detectors," Appl. Phys. Lett. 87(21), 211104 (2005).

12. J. Wang, J. Hu, X. Sun, A. Agarwal, and L. C. Kimerling, "Cavity-enhanced multispectral photodetector using phase-tuned propagation: theory and design," Opt. Lett. 35(5), 742-744 (2010).

13. X. C. Sun, J. J. Hu, C. Y. Hong, J. F. Viens, X. M. Duan, R. Das, A. M. Agarwal, and L. C. Kimerling, "Multispectral pixel performance using a one-dimensional photonic crystal design," Appl. Phys. Lett. 89(22), 223522 (2006).

14. M. Hammer, K. R. Hiremath, and R. Stoffer, "Analytical approaches to the description of Optical Microresonator Devices," in Microresonators as Building Blocks for VLSI Photonics, F. Michelotti, A. Driessen, and M.

Bertolotti, eds. (AIP Conference Proceedings, Melville, New York, 2004).

15. R. Swanepoel, "Determination of the thickness and optical constants of amorphous silicon," J. Phys. E 16(12), 1214-1222 (1983).

16. J. Wang, J. Hu, X. Sun, A. M. Agarwal, L. C. Kimerling, D. R. Lim, and R. A. Synowicki, "Structural, electrical, and optical properties of thermally evaporated nanocrystalline PbTe films," J. Appl. Phys. 104(5), 053707 (2008).

17. J. Hu, V. Tarasov, N. Carlie, L. Petit, A. Agarwal, K. Richardson, and L. C. Kimerling, "Exploration of Waveguide Fabrication From Thermally Evaporated Ge-Sb-S Glass Films," Opt. Mater. 30(10), 1560-1566 (2008). 